\title{
œcreative
}

\section{On hypohamiltonian snarks and a theorem of Fiorini*}

\author{
Jan Goedgebeur, Carol T. Zamfirescu \\ Department of Applied Mathematics, Computer Science \& Statistics, \\ Ghent University, Krijgslaan 281-S9, 9000 Ghent, Belgium \\ In loving memory of Ella.
}

Received 10 August 2016, accepted 1 May 2017, published online 4 September 2017

\begin{abstract}
In 2003, Cavicchioli et al. corrected an omission in the statement and proof of Fiorini's theorem from 1983 on hypohamiltonian snarks. However, their version of this theorem contains an unattainable condition for certain cases. We discuss and extend the results of Fiorini and Cavicchioli et al. and present a version of this theorem which is more general in several ways. Using Fiorini's erroneous result, Steffen had shown that hypohamiltonian snarks exist for some orders $n \geq 10$ and each even $n \geq 92$. We rectify Steffen's proof by providing a correct demonstration of a technical lemma on flower snarks, which might be of separate interest. We then strengthen Steffen's theorem to the strongest possible form by determining all orders for which hypohamiltonian snarks exist. This also strengthens a result of Máčajová and Škoviera. Finally, we verify a conjecture of Steffen on hypohamiltonian snarks up to 36 vertices.
\end{abstract}

Keywords: Hypohamiltonian, snark, irreducible snark, dot product.

Math. Subj. Class.: 05C10, 05C38, 05C45, 05C85

\section{Introduction}

A graph $G$ is hypohamiltonian if $G$ itself is non-hamiltonian, but for every vertex $v$ in $G$, the graph $G-v$ is hamiltonian. A snark shall be a cubic cyclically 4-edge-connected graph

* We thank Nico Van Cleemput for providing us with a script which greatly enhanced the quality of our figures. We would also like to thank Martin Škoviera for informing us about the equivalence of irreducible and vertexcritical graphs. Finally, we also wish to thank Eckhard Steffen for useful suggestions. Both authors are supported by a Postdoctoral Fellowship of the Research Foundation Flanders (FWO).

E-mail addresses: jan.goedgebeur@ugent.be (Jan Goedgebeur), czamfirescu@gmail.com (Carol T. Zamfirescu) 
with chromatic index 4 (i.e. four colours are required in any proper edge-colouring) and girth at least 5. We refer for notions not defined here to [22] and [7].

Motivated by similarities between the family of all snarks and the family of all cubic hypohamiltonian graphs regarding the orders for which such graphs exist, Fiorini [8] studied the hypohamiltonian properties surrounding Isaacs' so-called "flower snarks" [13] (defined rigorously below). The a priori surprising interplay between snarks and hypohamiltonian graphs has been investigated extensively-we now give an overview. Early contributions include Fiorini's aforementioned paper [8], in which he claims to show that there exist infinitely many hypohamiltonian snarks. (In fact, according to Máčajová and Škoviera [18], it was later discovered that a family of hypohamiltonian graphs constructed by Gutt [12] includes Isaacs' snarks, thus including Fiorini's result.)

Skupień showed that there exist exponentially many hypohamiltonian snarks [20], and Steffen [22] proved that there exist hypohamiltonian snarks of order $n$ for every even $n \geq 92$ (and certain $n<92$ )—we will come back to this result in Section 3. For more references and connections to other problems, see e.g. [3, 18, 20, 23]. Hypohamiltonian snarks have also been studied in connection with the famous Cycle Double Cover Conjecture [3] and Sabidussi's Compatibility Conjecture [9].

The smallest snark, as well as the smallest hypohamiltonian graph, is the famous Petersen graph. Steffen [21] showed that every cubic hypohamiltonian graph with chromatic index 4 is bicritical, i.e. the graph itself is not 3-edge-colourable but deleting any two distinct vertices yields a 3-edge-colourable graph. Nedela and Škoviera [19] proved that every cubic bicritical graph is cyclically 4-edge-connected and has girth at least 5. Therefore, every cubic hypohamiltonian graph with chromatic index 4 must be a snark.

This article is organised as follows. In Section 2 we discuss the omission in Fiorini's theorem on hypohamiltonian snarks [8] - first observed by Cavicchioli et al. [5] —and its consequences and state a more general version of this theorem. In Section 3 we first rectify a proof of Steffen on the orders for which hypohamiltonian snarks exist which relied on Fiorini's theorem - this erratum is based on giving a correct proof of a technical lemma concerning flower snarks, which may be of separate interest. We then prove a strengthening of Steffen's theorem, which is best possible, as all orders for which hypohamiltonian snarks exist are determined. Our result is stronger than a theorem of Máčajová and Škoviera [17] in the sense that our result implies theirs, while the converse does not hold. Finally, in Section 4 we comment upon and verify a conjecture of Steffen on hypohamiltonian snarks [23] for small orders.

\section{Fiorini's theorem revisited}

We call two edges independent if they have no common vertices. Let $G$ and $H$ be disjoint connected graphs on at least 6 vertices. Consider $G^{\prime}=G-\{a b, c d\}$, where $a b$ and $c d$ are independent edges in $G$, put $H^{\prime}=H-\{x, y\}$, where $x$ and $y$ are adjacent cubic vertices in $H$, and let $a^{\prime}, b^{\prime}$ and $c^{\prime}, d^{\prime}$ be the other neighbours of $x$ and $y$ in $H$, respectively. Then the dot product $G \cdot H$ is defined as the graph

$$
\left(V(G) \cup V\left(H^{\prime}\right), E\left(G^{\prime}\right) \cup E\left(H^{\prime}\right) \cup\left\{a a^{\prime}, b b^{\prime}, c c^{\prime}, d d^{\prime}\right\}\right) .
$$

Two remarks are in order. (1) Under the above conditions, the dot product may be disconnected. (2) In fact, there are eight ways to form the dot product for a specific $a b, c d, x y$. For the computational results in Section 4 we indeed applied the dot product in all eight 
possible ways, but for the theoretical proofs in this paper we will perform the dot product in one way, namely as follows. We always construct the dot product by adding the edges $a a^{\prime}, b b^{\prime}, c c^{\prime}, d d^{\prime}$ and we will abbreviate this as " $a, b, c, d$ are joined by edges to the neighbours of $x$ and $y$, respectively".

The dot product was introduced by Adelson-Velsky and Titov [1], and later and independently by Isaacs [13]. Its original purpose was to obtain new snarks by combining known snarks. Fiorini then proved that the dot product can also be used to combine two hypohamiltonian snarks into a new one. Unfortunately, Fiorini's argument is incorrect. We shall discuss this omission within this section, and correct the proof of a lemma of Steffen [22] which depended on Fiorini's result in Section 3.

In a graph $G$, a pair $(v, w)$ of vertices is good in $G$ if there exists a hamiltonian path in $G$ with end-vertices $v$ and $w$. Two pairs of vertices $((v, w),(x, y))$ are good in $G$ if there exist two disjoint paths which together $\operatorname{span} G$, and which have end-vertices $v$ and $w$, and $x$ and $y$, respectively.

Claim 2.1 (Fiorini, Theorem 3 in [8]). Let $G$ be a hypohamiltonian snark having two independent edges $a b$ and $c d$ for which

(i) each of $(a, c),(a, d),(b, c),(b, d),((a, b),(c, d))$ is good in $G$;

(ii) for each vertex $v$, exactly one of $(a, b),(c, d)$ is good in $G-v$.

If $H$ is a hypohamiltonian snark with adjacent vertices $x$ and $y$, then the dot product $G \cdot H$ is also a hypohamiltonian snark, where ab and cd are deleted from $G, x$ and $y$ are deleted from $H$, and vertices $a, b, c, d$ are joined by edges to the neighbours of $x$ and $y$, respectively.

Cavicchioli et al. [5] point out the omissions in Claim 2.1: in order for the proof to work, the given vertex pairs need to be good in $G-\{a b, c d\}$ rather than in $G$. They give a corrected statement of the theorem envisioned by Fiorini and give a new proof.

Claim 2.2 (Cavicchioli et al., Theorem 3.2 in [5]). Let $G$ be a hypohamiltonian snark having two independent edges ab and cd for which

(i) each of $(a, c),(a, d),(b, c),(b, d),((a, b),(c, d))$ is good in $G-\{a b, c d\}$;

(ii) for each vertex $v$, each of $(a, b),(c, d)$ is good in $G-\{v, a b, c d\}$.

If $H$ is a hypohamiltonian snark with adjacent vertices $x$ and $y$, then the dot product $G \cdot H$ is also a hypohamiltonian snark, where $a b$ and $c d$ are deleted from $G, x$ and $y$ are deleted from $H$, and vertices $a, b, c, d$ are joined by edges to the neighbours of $x$ and $y$, respectively.

In above statements, the fact that the dot product of snarks is itself a snark had already been shown [1, 13], so indeed only the hypohamiltonicity was to be proven.

We point out that the hypotheses in Claim 2.2 are unattainable for $v \in\{a, b, c, d\}$, since $(a, b)$ and $(c, d)$ cannot both be good in $G-\{v, a b, c d\}$ if $v \in\{a, b, c, d\}$. This is tied to the fact that the requirements in (ii) are stronger than what is needed to prove the statement.

In [11, Theorem 1], we gave the following (second) restatement of Claim 2.1 which we used to solve a problem of McKay. Note that in [11] the graphs are required to be cubic and below we do not state this requirement-we do however need the two vertices which are removed to be cubic. This allows us to use exactly the same proof as in [11, Theorem 1]. Nevertheless, we now give a sketch of the proof: first, we assume that $G \cdot H$ does contain a hamiltonian cycle. This however implies that at least one of the factors is hamiltonian, 
contradicting their hypohamiltonicity. Second, we prove that every vertex-deleted subgraph of $G \cdot H$ is indeed hamiltonian. This is done with a careful case analysis (depending on where the removed vertex lies) using the goodness of various pairs (and pairs of pairs) of vertices in $G-\{a b, c d\}$ and $G-\{v, a b, c d\}$.

Theorem 2.3. Let $G$ be a non-hamiltonian graph having two independent edges ab and $c d$ for which

(i) each of $(a, c),(a, d),(b, c),(b, d),((a, b),(c, d))$ is good in $G-\{a b, c d\}$;

(ii) for each vertex $v$, at least one of $(a, b)$ and $(c, d)$ is good in $G-\{v, a b, c d\}$.

If $H$ is a hypohamiltonian graph with cubic adjacent vertices $x$ and $y$, then the dot product $G \cdot H$ is also a hypohamiltonian graph, where $a b$ and $c d$ are deleted from $G, x$ and $y$ are deleted from $H$, and vertices $a, b, c, d$ are joined by edges to the neighbours of $x$ and $y$, respectively.

If $G$ and $H$ are planar, and $a b$ and cd lie on the same facial cycle, then the dot product can be applied such that $G \cdot H$ is planar, as well. If $g$ and $h$ are the girth of $G$ and $H$, respectively, then the girth of $G \cdot H$ is at least $\min \{g, h\}$. If $G$ and $H$ are cubic, then so is $G \cdot H$.

Note that the fact that $G$ is non-hamiltonian together with condition (ii) implies that $G$ must be hypohamiltonian.

In the following, we will call the pair of edges $a b, c d$ from the statement of Theorem 2.3 suitable. The Petersen graph is the smallest snark, and the two Blanuša snarks on 18 vertices are the second-smallest snarks. All three graphs are also hypohamiltonian. Due to the huge automorphism group of the Petersen graph, it can be verified by hand that it does not contain a pair of suitable edges. Although both Blanuša snarks are dot products of two Petersen graphs, the Petersen graph does not contain a pair of suitable edges. Thus, in a certain sense, Theorem 2.3 is not "if and only if", i.e. there exist dot products whose factors do not contain suitable edges.

Let us end this section with a remark which may prove to be useful in other applications. Throughout its statement and proof, we use the notation from Theorem 2.3.

Observation 2.4. We have that $G \cdot H+a b, G \cdot H+c d$, and $G \cdot H+a b+c d$ are hypohamiltonian, as well.

Proof. Put $N(x)=\left\{a^{\prime}, b^{\prime}, y\right\}$ and $N(y)=\left\{c^{\prime}, d^{\prime}, x\right\}$ such that the unique neighbour of $a^{\prime}\left(b^{\prime}, c^{\prime}, d^{\prime}\right)$ in $G$ is $a(b, c, d)$. Assume $G \cdot H+a b+c d$ contains a hamiltonian cycle $\mathfrak{h}$. Thus, at least one of $a b$ and $c d$ lies in $\mathfrak{h}$, say $a b$. We treat $H-\{x, y\}$ as a subgraph of $G \cdot H$. If $a a^{\prime}, b b^{\prime} \in E(\mathfrak{h})$, then $\mathfrak{h} \cap H \cup a^{\prime} x b^{\prime} \cup c^{\prime} y d^{\prime}$ gives a hamiltonian cycle in $H$, a contradiction. If $a a^{\prime}, b b^{\prime} \notin E(\mathfrak{h})$, then the cycle $\mathfrak{h} \cap G+c d$ yields a contradiction. So w.l.o.g. $a a^{\prime} \in E(\mathfrak{h})$ and $b b^{\prime} \notin E(\mathfrak{h})$. This implies the existence of a hamiltonian path in $H-\{x, y\}$ with end-vertices $a^{\prime}$ and $u \in\left\{c^{\prime}, d^{\prime}\right\}$. But this path together with $u y x a^{\prime}$ is a hamiltonian cycle in $H$, a contradiction. It follows that $G \cdot H+a b$ and $G \cdot H+c d$ are non-hamiltonian, as well.

\section{On a theorem of Steffen on hypohamiltonian snarks}

\subsection{Rectifying Steffen's proof}

A snark is irreducible if the removal of every edge-cut which is not the set of all edges incident with a vertex yields a 3-edge-colourable graph. Steffen's article [22] is motivated 
by the following problem.

Problem 3.1 (Nedela and Škoviera [19]). For which even number $n \geq 10$ does there exist an irreducible snark of order $n$ ? In particular, does there exist an irreducible snark of each sufficiently large order?

Steffen settled the second question of Problem 3.1 by giving the following main result from [22].

Theorem 3.2 (Steffen, Theorem 2.5 in [22]). There is a hypohamiltonian snark of order $n$

(1) for each $n \in\{m: m \geq 64$ and $m \equiv 0 \bmod 8\}$,

(2) for each $n \in\{10,18\} \cup\{m: m \geq 98$ and $m \equiv 2 \bmod 8\}$,

(3) for each $n \in\{m: m \geq 20$ and $m \equiv 4 \bmod 8\}$,

(4) for each $n \in\{30\} \cup\{m: m \geq 54$ and $m \equiv 6 \bmod 8\}$, and

(5) for each even $n \geq 92$.

Isaacs' flower snark $J_{2 k+1}$, see [13], is the graph

$$
\left(\left\{a_{i}, b_{i}, c_{i}, d_{i}\right\}_{i=0}^{2 k},\left\{b_{i} a_{i}, b_{i} c_{i}, b_{i} d_{i}, a_{i} a_{i+1}, c_{i} d_{i+1}, d_{i} c_{i+1}\right\}_{i=0}^{2 k}\right),
$$

where addition in the indices is performed modulo $2 k+1$.

However, the proof of [22, Lemma 2.3], which is essential for the proof of the theorem, is erroneous, since it uses Fiorini's erroneous Claim 2.1 (and it does not work with Theorem 2.3). We here give a correct proof of that lemma.

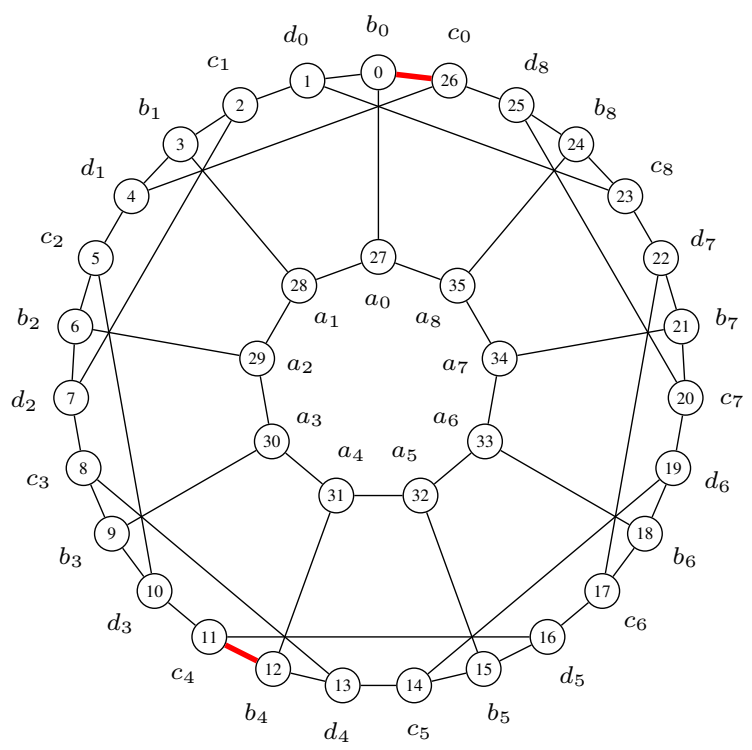

Figure 1: The flower snark $J_{9}$. The suitable edges $b_{0} c_{0}$ and $b_{4} c_{4}$ are marked in bold red. 
Lemma 3.3 (Steffen, Lemma 2.3 in [22]). The flower snarks $J_{9}, J_{11}$, and $J_{13}$ satisfy the conditions of Theorem 2.3.

Proof. In [22], in each of the graphs $J_{9}, J_{11}$, and $J_{13}$, the suitable edges were chosen to be $b_{0} c_{0}$ and $b_{4} c_{4}$. However, in [22], for various vertices $v$, the hamiltonian paths did not satisfy condition (ii) from Theorem 2.3, as the paths used one of the edges $b_{0} c_{0}$ or $b_{4} c_{4}$. This was for instance the case for $v \in\left\{a_{0}, a_{8}, d_{0}\right\}$ in $J_{9}$, for $v \in\left\{a_{0}, a_{10}, d_{0}\right\}$ in $J_{11}$, and for $v \in\left\{a_{0}, c_{1}, c_{12}, d_{0}\right\}$ in $J_{13}$, see Claims 6, 7, and 8 in the Appendix of [22].

We will now prove that $b_{0} c_{0}$ and $b_{4} c_{4}$ are indeed suitable edges for Theorem 2.3 for $J_{9}$, $J_{11}$, and $J_{13}$. For $J_{9}$ the proof is given below (and partially in the Appendix), while the technical details of the proofs for $J_{11}$ and $J_{13}$ can be found in the Appendix. The mapping between the $a_{i}, b_{i}, c_{i}, d_{i}$ (used by Steffen) and the vertex numbers used in the proof is shown in Figures 1-3. We use numbers as labels in the proof to make it easier to read these graphs using a computer for verifying the results.

Proof that $\boldsymbol{b}_{\mathbf{0}} \boldsymbol{c}_{\mathbf{0}}$ and $\boldsymbol{b}_{\mathbf{4}} \boldsymbol{c}_{\mathbf{4}}$ are suitable edges for $\boldsymbol{J}_{\mathbf{9}}$. Figure 1 shows the flower snark $J_{9}$. In $J_{9}$, the edges $b_{0} c_{0}$ and $b_{4} c_{4}$ correspond to the edges $(0,26)$ and $(11,12)$, respectively.

The pairs $(0,11),(0,12),(26,11)$ and $(26,12)$ are good in $J_{9}-\{(0,26),(11,12)\}$ due to the following hamiltonian paths, respectively:

- $11,10,5,6,7,8,9,30,29,28,27,35,24,23,22,17,16,15,32,31,12,13,14,19$, $18,33,34,21,20,25,26,4,3,2,1,0$

- $12,13,14,15,16,11,10,5,4,26,25,20,19,18,17,22,21,34,33,32,31,30,9,8$, $7,6,29,28,3,2,1,23,24,35,27,0$

- $11,10,5,4,3,2,1,0,27,28,29,6,7,8,9,30,31,12,13,14,19,18,17,16,15,32$, $33,34,35,24,23,22,21,20,25,26$

- $12,13,8,7,2,3,4,5,6,29,28,27,0,1,23,22,17,18,33,32,31,30,9,10,11,16$, $15,14,19,20,21,34,35,24,25,26$

Note that $((0,26),(11,12))$ is good in $J_{9}-\{(0,26),(11,12)\}$ due to the following two disjoint paths with end-vertices 0 and 26 , and 11 and 12 , respectively, which together $\operatorname{span} J_{9}$ :

- $26,25,20,19,14,13,8,7,2,1,0$

- $12,31,32,15,16,17,18,33,34,21,22,23,24,35,27,28,3,4,5,6,29,30,9,10$, 11

We showed by computer that at least one of $(0,26)$ or $(11,12)$ is good in $J_{9}-\{v,(0,26)$, $(11,12)\}$ for every $v \in V\left(J_{9}\right)$. In each case we verified that the path found by the computer is indeed a valid hamiltonian path in the graph. Below we explicitly show this for $v=0$. The hamiltonian paths for the other choices of $v$ can be found in the Appendix.

- $v=0: 12,13,14,15,32,31,30,29,6,5,10,9,8,7,2,1,23,24,25,26,4,3,28$, $27,35,34,33,18,19,20,21,22,17,16,11$

Since Steffen's statement of Lemma 3.3 remains intact, the proof and statement of his main result, reproduced above as Theorem 3.2, are correct as given in [22]. Even though we prove a stronger version of Steffen's theorem in the next section, we think it is important to fix the proof of Lemma 3.3 as there may be others who rely on this lemma, or might want to rely on it in the future. 


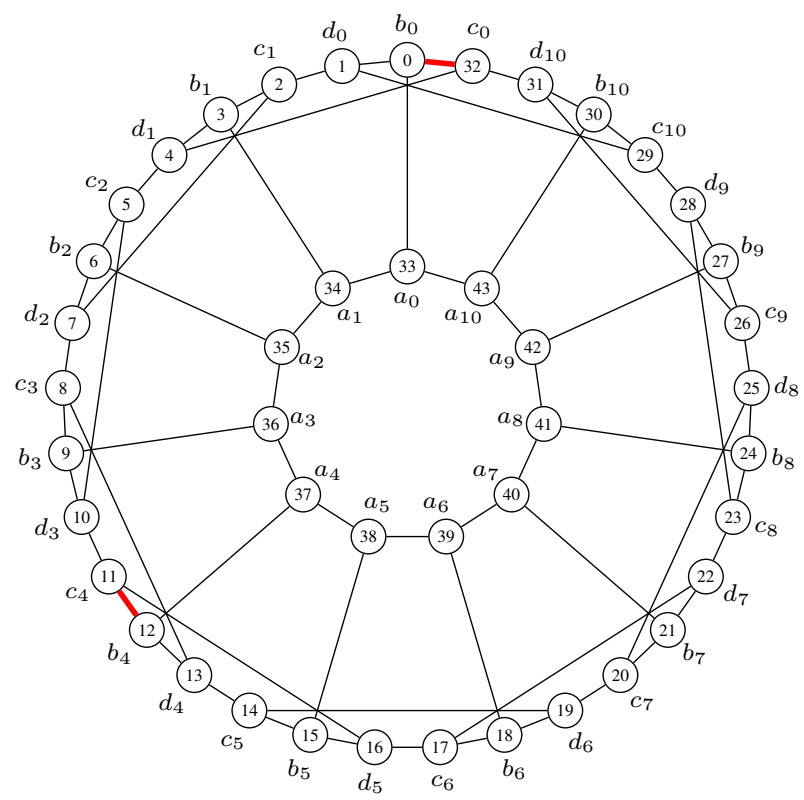

Figure 2: The flower snark $J_{11}$. The suitable edges $b_{0} c_{0}$ and $b_{4} c_{4}$ are marked in bold red.

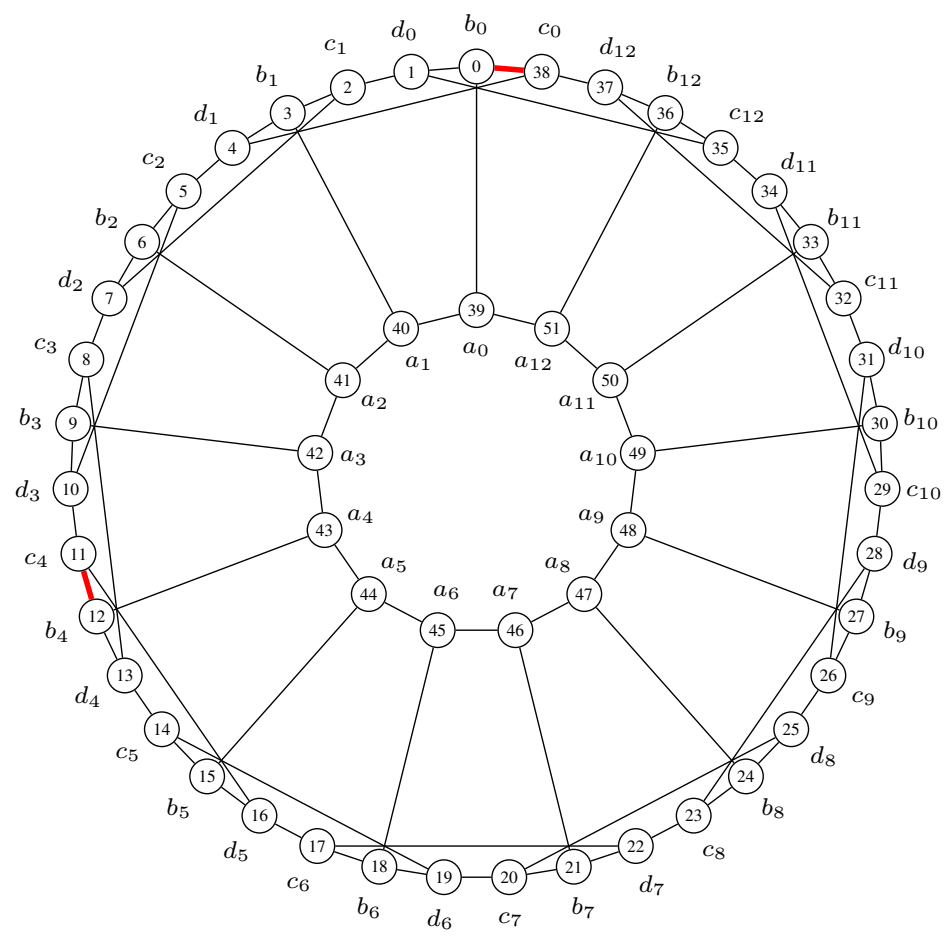

Figure 3: The flower snark $J_{13}$. The suitable edges $b_{0} c_{0}$ and $b_{4} c_{4}$ are marked in bold red. 


\subsection{Orders of hypohamiltonian snarks}

We shall now prove a strengthening of Steffen's theorem, which in a sense is strongest possible since we will determine all orders for which hypohamiltonian snarks exist. We emphasise that our proof's mechanism contains significantly fewer "moving parts" than Máčajová and Škoviera's [17], and, as mentioned in the introduction, our theorem also strengthens their result. We do need the following two easily verifiable lemmas.

Lemma 3.4. The second Blanuša snark $B_{2}$ shown in Figure 4 has a pair of suitable edges.

Proof. Figure 4 shows the second Blanuša snark $B_{2}$. By computer we determined that $B_{2}$ has exactly three pairs of suitable edges: $((6,8),(10,16)),((3,9),(12,17))$ and $((4,7)$, $(13,15))$. We will now prove by hand that $((6,8),(10,16))$ is a suitable edge pair.

The pairs $(6,10),(6,16),(8,10)$ and $(8,16)$ are good in $B_{2}-\{(6,8),(10,16)\}$ due to the following hamiltonian paths, respectively:

- $10,11,12,17,16,15,13,14,0,1,5,4,7,8,9,3,2,6$

- $16,15,9,8,7,17,12,13,14,10,11,1,0,2,3,4,5,6$

- $10,11,1,0,14,13,12,17,16,15,9,3,2,6,5,4,7,8$

- $16,15,9,3,4,5,6,2,0,1,11,10,14,13,12,17,7,8$

Note that $((6,8),(10,16))$ is good in $B_{2}-\{(6,8),(10,16)\}$ due to the following two disjoint paths with end-vertices 6 and 8 , and 10 and 16, respectively, which together span $B_{2}$ :

- $8,7,4,5,6$

- $16,17,12,11,1,0,2,3,9,15,13,14,10$

We now prove that at least one of $(6,8)$ or $(10,16)$ is good in $B_{2}-\{v,(6,8),(10,16)\}$ for every $v \in V\left(B_{2}\right)$. By symmetry we only need to prove this for $v=0,2,4,6,7,8$.

- $v=0: 8,7,4,5,1,11,10,14,13,12,17,16,15,9,3,2,6$

- $v=2: 8,9,3,4,7,17,16,15,13,12,11,10,14,0,1,5,6$

- $v=4: 16,15,13,14,0,1,5,6,2,3,9,8,7,17,12,11,10$

- $v=6: 16,15,9,8,7,17,12,13,14,0,2,3,4,5,1,11,10$

- $v=7: 8,9,15,16,17,12,13,14,10,11,1,0,2,3,4,5,6$

- $v=8: 16,15,9,3,2,6,5,4,7,17,12,13,14,0,1,11,10$

Lemma 3.5. The first Loupekine snark $L_{1}$ shown in Figure 5 has a pair of suitable edges.

Proof. Figure 5 shows the first Loupekine snark $L_{1}$. By computer we determined that $L_{1}$ has exactly six pairs of suitable edges: $((0,1),(17,20)),((0,2),(8,17)),((1,5),(14,20))$, $((2,3),(8,10)),((3,4),(10,12))$ and $((4,5),(12,14))$. We will now prove by hand that $((3,4),(10,12))$ is a suitable edge pair.

The pairs $(3,10),(3,12),(4,10)$ and $(4,12)$ are good in $L_{1}-\{(3,4),(10,12)\}$ due to the following hamiltonian paths, respectively:

- $10,7,9,6,8,17,19,21,16,13,11,0,1,5,4,18,20,14,12,15,2,3$ 


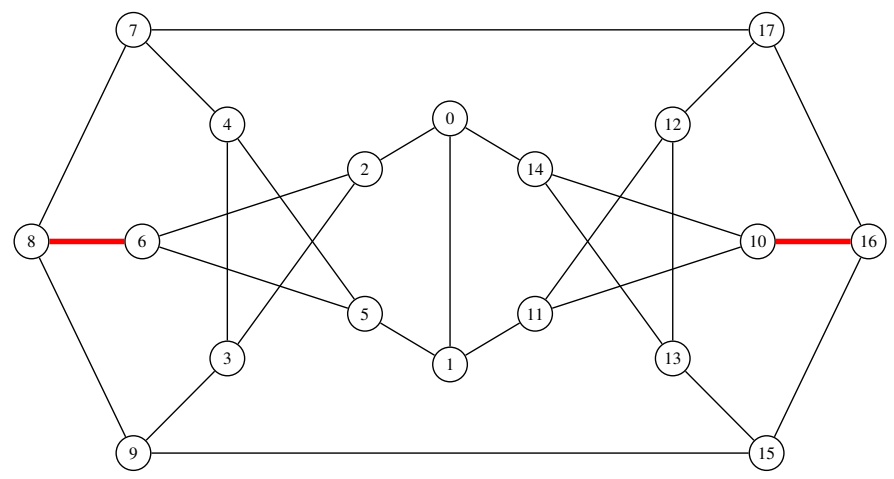

Figure 4: The second Blanuša snark. It has 18 vertices. The suitable edges $(6,8)$ and $(10,16)$ are marked in bold red.

- $12,14,20,18,4,5,7,10,8,17,19,21,16,9,6,1,0,11,13,15,2,3$

- $10,7,5,1,0,11,13,16,9,6,8,17,20,14,12,15,2,3,19,21,18,4$

- $12,14,11,0,1,6,9,16,13,15,2,3,19,21,18,20,17,8,10,7,5,4$

Note that $((3,4),(10,12))$ is good in $L_{1}-\{(3,4),(10,12)\}$ due to the following two disjoint paths with end-vertices 3 and 4 , and 10 and 12 , respectively, which together span $L_{1}$ :

- $4,5,1,6,8,17,20,18,21,19,3$

- $12,14,11,0,2,15,13,16,9,7,10$

We now prove that at least one of $(3,4)$ or $(10,12)$ is good in $L_{1}-\{v,(3,4),(10,12)\}$ for every $v \in V\left(L_{1}\right)$. By symmetry we only need to prove this for $v=1,4,5,6,7,8,9$, $10,16,17,18,21$.

- $v=1: 12,15,13,16,9,6,8,17,20,14,11,0,2,3,19,21,18,4,5,7,10$

- $v=4: 12,14,20,18,21,16,9,6,8,17,19,3,2,15,13,11,0,1,5,7,10$

- $v=5: 4,18,20,17,8,10,7,9,6,1,0,2,15,12,14,11,13,16,21,19,3$

- $v=6: 4,5,1,0,2,15,12,14,11,13,16,9,7,10,8,17,20,18,21,19,3$

- $v=7: 12,14,20,17,19,3,2,15,13,11,0,1,5,4,18,21,16,9,6,8,10$

- $v=8: 12,14,20,17,19,3,2,15,13,11,0,1,6,9,16,21,18,4,5,7,10$

- $v=9: 4,5,7,10,8,6,1,0,2,15,12,14,11,13,16,21,18,20,17,19,3$

- $v=10: 4,5,7,9,16,13,11,0,1,6,8,17,19,21,18,20,14,12,15,2,3$

- $v=16: 4,5,1,6,9,7,10,8,17,19,21,18,20,14,12,15,13,11,0,2,3$

- $v=17: 4,5,1,6,8,10,7,9,16,13,11,0,2,15,12,14,20,18,21,19,3$

- $v=18: 4,5,1,6,8,10,7,9,16,21,19,17,20,14,12,15,13,11,0,2,3$

- $v=21: 4,18,20,14,12,15,2,0,11,13,16,9,6,1,5,7,10,8,17,19,3$ 


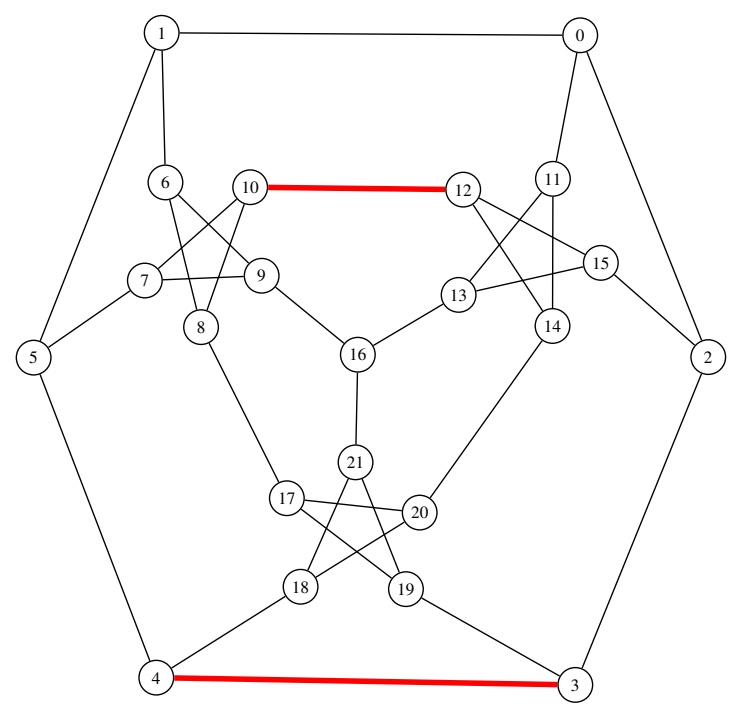

Figure 5: The first Loupekine snark $L_{1}$. It has 22 vertices. The suitable edges $(3,4)$ and $(10,12)$ are marked in bold red.

The following generalisation of Steffen's Theorem 3.2 is strongest possible.

Theorem 3.6. A hypohamiltonian snark of order $n$ exists if and only if $n \in\{10,18,20,22\}$ or $n$ is even and $n \geq 26$.

Proof. For $n=10$, it is well-known that the Petersen graph is hypohamiltonian and it is also well-known that no snarks exist of order 12, 14 or 16.

In Lemma 3.4 we showed that the second Blanuša snark $B_{2}$ (which has order 18) contains a pair of suitable edges. In [3] it was proven that hypohamiltonian snarks exist for all even orders from 18 to 36 with the exception of 24 (see Table 1). Let $S_{n}$ denote a hypohamiltonian snark of order $n$. Using Theorem 2.3, we form the dot product $B_{2} \cdot S_{n}$ for $n \in\{18,20,22,26,28,30,32\}$ and obtain hypohamiltonian snarks of all even orders between 34 and 48 with the exception of 40 (recall that the dot product of two snarks is a snark).

By Lemma 3.5 we know that the first Loupekine snark $L_{1}$ (which has order 22) contains a pair of suitable edges. Applying Theorem 2.3 to this snark and a 22-vertex hypohamiltonian snark, we obtain a hypohamiltonian snark of order 40 .

We form the dot product $B_{2} \cdot S_{n}$ for all even $n \in\{34, \ldots, 48\}$ and obtain hypohamiltonian snarks of all even orders from 50 to 64 . This may now be iterated ad infinitum, and the proof is complete.

\subsection{Hypohamiltonian and irreducible snarks}

In [17] Máčajová and Škoviera proved the following theorem (which fully settles Problem 3.1).

Theorem 3.7 (Máčajová and Škoviera, Theorems A and B in [17]). There exists an irreducible snark of order $n$ if and only if $n \in\{10,18,20,22\}$ or $n$ is even and $n \geq 26$. 
Nedela and Škoviera [19] proved that a snark is irreducible if and only if it is bicritical, and Steffen [21] showed that every hypohamiltonian snark is bicritical-while the converse is not true, as will be shown in Table 1.

A graph $G$ without $k$-flow is $k$-vertex-critical if, for every pair of vertices $(u, v)$ of $G$, identifying $u$ and $v$ yields a graph that has a $k$-flow; see [4] for more details. In [4] Carneiro, da Silva, and McKay determined all 4-vertex-critical snarks up to 36 vertices and Škoviera [24] showed that a snark is 4-vertex-critical if and only if it is irreducible.

Cavicchioli et al. [5] determined all hypohamiltonian and irreducible snarks on $n \leq 28$ vertices. Later, Brinkmann et al. [3] determined all hypohamiltonian snarks on $n \leq 36$ vertices. These counts can be found in Table 1 together with the number of irreducible snarks from [4]. These graphs can also be downloaded from the House of Graphs [2] at http://hog.grinvin.org/Snarks.

The number of hypohamiltonian cubic graphs on $n \leq 32$ vertices can be found in [10]. As can be seen from Table 1, there is a significant number of irreducible snarks which are not hypohamiltonian. The smallest such snarks have order 26. So Theorem 3.6 implies Theorem 3.7, while the converse is not true.

Table 1: Number of irreducible and hypohamiltonian snarks (see [4, Table 1] and [3, Table 2]). $\lambda_{c}$ stands for cyclic edge-connectivity. The counts of cases indicated with a ' $\geq$ ' are possibly incomplete; all other cases are complete.

\begin{tabular}{|c|r|r|r|r|}
\hline Order & irreducible & hypo. & hypo. and $\lambda_{c}=4$ & hypo. and $\lambda_{c} \geq 5$ \\
\hline 10 & 1 & 1 & 0 & 1 \\
18 & 2 & 2 & 2 & 0 \\
20 & 1 & 1 & 0 & 1 \\
22 & 2 & 2 & 0 & 2 \\
24 & 0 & 0 & 0 & 0 \\
26 & 111 & 95 & 87 & 8 \\
28 & 33 & 31 & 30 & 1 \\
30 & 115 & 104 & 93 & 11 \\
32 & 13 & 13 & 0 & 13 \\
34 & 40328 & 31198 & 29701 & 1497 \\
36 & 13720 & 10838 & 10374 & 464 \\
38 & $?$ & $?$ & $\geq 51431$ & $?$ \\
40 & $?$ & $?$ & $\geq 8820$ & $?$ \\
42 & $?$ & $?$ & $\geq 20575458$ & $?$ \\
44 & $?$ & $?$ & $\geq 8242146$ & $?$ \\
\hline
\end{tabular}

The hypohamiltonian snarks on $n \geq 34$ vertices constructed by the dot product in the proof of Theorem 3.6 clearly all have cyclic edge-connectivity 4 . By combining this with Table 1 we obtain:

Corollary 3.8. Hypohamiltonian snarks of order $n$ and cyclic edge-connectivity 4 exist if and only if $n \in\{18,26,28,30\}$ or $n$ is even and $n \geq 34$.

As already mentioned, every hypohamiltonian snark is irreducible, thus Corollary 3.8 implies [17, Theorem A]. For higher cyclic edge-connectivity, the following is known. 
Theorem 3.9 (Máčajová and Škoviera [18]). There exists a hypohamiltonian snark of order $n$ and cyclic connectivity 5 for each even $n \geq 140$, and there exists a hypohamiltonian snark of order $n$ and cyclic connectivity 6 for each even $n \geq 166$.

If we relax the requirements from hypohamiltonicity to irreducibility, more is known:

Theorem 3.10 (Máčajová and Škoviera [17]). There exists an irreducible snark of order $n$ and cyclic connectivity 5 if and only if $n \in\{10,20,22,26\}$ or $n$ is even and $n \geq 30$, and there exists an irreducible snark of order $n$ and cyclic connectivity 6 for each $n \equiv 4(\bmod 8)$ with $n \geq 28$, and for each even $n \geq 210$.

Note that as every hypohamiltonian snark is irreducible, Theorem 3.9 also implies that $n \geq 210$ can be improved to $n \geq 166$ in Theorem 3.10 .

The smallest hypohamiltonian snark of cyclic edge-connectivity 5 has order 10 and is the Petersen graph, and the second-smallest such graph has order 20 . The flower snark $J_{7}$ of order 28 is the smallest cyclically 6-edge-connected hypohamiltonian snark. We conclude this section with the following two problems motivated by Theorem 3.10 and results of Kochol [14, 16].

Problem 3.11 (Máčajová and Škoviera [17]). Construct a cyclically 6-edge-connected snark (irreducible or not) of order smaller than 118 and different from any of Isaacs' snarks.

Problem 3.12. Determine all orders for which cyclically 6 -edge-connected snarks exist.

\section{On a conjecture of Steffen on hypohamiltonian snarks}

Consider a cubic graph $G$. We denote with $\mu_{k}(G)$ the minimum number of edges not contained in the union of $k 1$-factors of $G$, for every possible combination of $k 1$-factors. If $\mu_{3}(G)=0$, then $G$ is 3-edge-colourable. In [23], Steffen made the following conjecture on hypohamiltonian snarks.

Conjecture 4.1 (Steffen, Conjecture 4.1 in [23]). If $G$ is a hypohamiltonian snark, then $\mu_{3}(G)=3$.

If true, this conjecture would have significant consequences, e.g. by Theorem 2.14 from [23], it would imply that every hypohamiltonian snark has a Berge-cover (a bridgeless cubic graph $G$ has a Berge-cover if $\mu_{5}(G)=0$ ).

We wrote a computer program for computing $\mu_{3}(G)$ and tested Conjecture 4.1 on the complete lists of hypohamiltonian snarks up to 36 vertices. This leads to the following observation.

Observation 4.2. There are no counterexamples to Conjecture 4.1 among the hypohamiltonian snarks with at most 36 vertices.

The authors of [3] noted a huge increase (from 13 to 31 198) in the number of hypohamiltonian snarks from order 32 to 34 , see Table 1 . Using a computer, we were able to determine that 29365 out of the 29701 hypohamiltonian snarks with cyclic edgeconnectivity 4 on 34 vertices can be obtained by performing a dot product on a hypohamiltonian snark on 26 vertices and the Petersen graph. We also determined that the remaining hypohamiltonian snarks with cyclic edge-connectivity 4 on 34 vertices are obtained by performing a dot product on the Blanuša snarks. Intriguingly, our computations show that 
some hypohamiltonian snarks can be obtained by performing a dot product on a hypohamiltonian snark on 26 vertices and the Petersen graph, as well as by performing a dot product on the Blanuša snarks.

There is also a (slightly less dramatic) increase in the cyclically 5-edge-connected case-these are obviously not dot products-and we believe it to be due to more general graph products, for instance "superposition" introduced by Kochol [15]. It would be interesting to further explore these transformations in order to fully understand these sudden increases and decreases in numbers.

Using a computer, we determined that all hypohamiltonian snarks with cyclic edgeconnectivity 4 up to 36 vertices can be obtained by performing a dot product on two hypohamiltonian snarks. This leads us to pose the following question.

Problem 4.3. Is every hypohamiltonian snark with cyclic edge-connectivity 4 a dot product of two hypohamiltonian snarks?

In [6] Chladný and Škoviera proved that every bicritical snark with cyclic edge-connectivity 4 is a dot product of two bicritical snarks. Since every hypohamiltonian snark is bicritical, this implies that every hypohamiltonian snark with cyclic edge-connectivity 4 is a dot product of two bicritical snarks.

Recall that in Theorem 2.3 the graphs $G$ and $H$ are hypohamiltonian, but the theorem is not "if and only if", since although the Petersen graph does not contain a pair of suitable edges, the Blanuša snarks (which are dot products of two Petersen graphs) are hypohamiltonian. Despite the previous paragraph, we believe the answer to Problem 4.3 to be "no" due to the following observation. In order to cover all cases, we would need to add to condition (ii) of Theorem 2.3 the possibility of $((a, b),(c, d))$ being good in $G-\{v, a b, c d\}$. However, we would then need to require from $H$ that it contains a 2-factor containing exactly two (necessarily odd) cycles. Although we were unable to find a counterexample, we believe that there exist hypohamiltonian snarks which do not possess such a 2-factor.

We also determined all hypohamiltonian snarks up to 44 vertices which can be obtained by performing a dot product on two hypohamiltonian snarks. The counts of these snarks can be found in the fourth column of Table 1. We also verified Conjecture 4.1 on these snarks.

Observation 4.4. There are no counterexamples to Conjecture 4.1 among the hypohamiltonian snarks with at most 44 vertices which are a dot product of two hypohamiltonian snarks.

\section{References}

[1] G. M. Adelson-Velsky and V. K. Titov, On edge 4-chromatic cubic graphs (in Russian), in: Proc. Seminar of 1971 at Moscow Univ., Voprosy Kibernetiki, 1973 pp. 5-14.

[2] G. Brinkmann, K. Coolsaet, J. Goedgebeur and H. Mélot, House of Graphs: a database of interesting graphs, Discrete Appl. Math. 161 (2013), 311-314, doi:10.1016/j.dam.2012.07.018, available at http://hog.grinvin.org/.

[3] G. Brinkmann, J. Goedgebeur, J. Hägglund and K. Markström, Generation and properties of snarks, J. Combin. Theory Ser. B 103 (2013), 468-488, doi:10.1016/j.jctb.2013.05.001.

[4] A. B. Carneiro, C. N. da Silva and B. D. McKay, A faster test for 4-flow-criticality in snarks, Electron. Notes Discrete Math. 50 (2015), 193-198, doi:10.1016/j.endm.2015.07.033. 
[5] A. Cavicchioli, T. E. Murgolo, B. Ruini and F. Spaggiari, Special classes of snarks, Acta Appl. Math. 76 (2003), 57-88, doi:10.1023/a:1022864000162.

[6] M. Chladný and M. Škoviera, Factorisation of snarks, Electron. J. Combin. 17 (2010), \#R32, http://www.combinatorics.org/ojs/index.php/eljc/article/view/ v17i1r32.

[7] R. Diestel, Graph Theory, volume 173 of Graduate Texts in Mathematics, Springer, Heidelberg, 4th edition, 2010, doi:10.1007/978-3-642-14279-6.

[8] S. Fiorini, Hypohamiltonian snarks, in: M. Fiedler (ed.), Graphs and Other Combinatorial Topics, Teubner, Leipzig, volume 59 of Teubner-Texte zur Mathematik, 1983 pp. 70-75.

[9] H. Fleischner and R. Häggkvist, Circuit double covers in special types of cubic graphs, Discrete Math. 309 (2009), 5724-5728, doi:10.1016/j.disc.2008.05.018.

[10] J. Goedgebeur and C. T. Zamfirescu, Improved bounds for hypohamiltonian graphs, Ars Math. Contemp. 13 (2017), 235-257, http://amc-journal.eu/index.php/amc/ article/view/1044.

[11] J. Goedgebeur and C. T. Zamfirescu, Infinitely many planar cubic hypohamiltonian graphs of girth 5, doi:10.1002/jgt.22183, to appear in J. Graph Theory.

[12] S. Gutt, Infinite families of hypohamiltonian graphs, Acad. Roy. Belg. Bull. Cl. Sci. 63 (1977), $432-440$.

[13] R. Isaacs, Infinite families of nontrivial trivalent graphs which are not Tait colorable, Amer. Math. Monthly 82 (1975), 221-239, doi:10.2307/2319844.

[14] M. Kochol, A cyclically 6-edge-connected snark of order 118, Discrete Math. 161 (1996), 297-300, doi:10.1016/0012-365x(95)00237-q.

[15] M. Kochol, Snarks without small cycles, J. Combin. Theory Ser. B 67 (1996), 34-47, doi: 10.1006/jctb.1996.0032.

[16] M. Kochol, Reduction of the 5-flow conjecture to cyclically 6-edge-connected snarks, J. Combin. Theory Ser. B 90 (2004), 139-145, doi:10.1016/s0095-8956(03)00080-7.

[17] E. Máčajová and M. Škoviera, Irreducible snarks of given order and cyclic connectivity, Discrete Math. 306 (2006), 779-791, doi:10.1016/j.disc.2006.02.003.

[18] E. Máčajová and M. Škoviera, Constructing hypohamiltonian snarks with cyclic connectivity 5 and 6, Electron. J. Combin. 14 (2007), \#R18, http: / /www. combinatorics.org/ojs / index.php/eljc/article/view/v14ilr18.

[19] R. Nedela and M. Škoviera, Decompositions and reductions of snarks, J. Graph Theory 22 (1996), 253-279, doi:10.1002/(sici)1097-0118(199607)22:3〈253::aid-jgt6〉3.0.co;2-1.

[20] Z. Skupień, Exponentially many hypohamiltonian snarks, Electron. Notes Discrete Math. 28 (2007), 417-424, doi:10.1016/j.endm.2007.01.059.

[21] E. Steffen, Classification and characterizations of snarks, Discrete Math. 188 (1998), 183-203, doi:10.1016/s0012-365x(97)00255-0.

[22] E. Steffen, On bicritical snarks, Math. Slovaca 51 (2001), 141-150.

[23] E. Steffen, 1-factor and cycle covers of cubic graphs, J. Graph Theory 78 (2015), 195-206, doi:10.1002/jgt.21798.

[24] M. Škoviera, Flow-critical and critical snarks coincide, 2016, in preparation. 


\section{Appendix}

Below we give the technical details which were left out in the proof of Lemma 3.3.

\section{Proof that $b_{0} c_{0}$ and $b_{4} c_{4}$ are suitable edges for $J_{9}$ (continued)}

We will now prove that at least one of $(0,26)$ or $(11,12)$ is good in $J_{9}-\{v,(0,26)$, $(11,12)\}$ for every $v \in V\left(J_{9}\right)$ except for $v=0$, which we have already shown above in the proof of Lemma 3.3.

- $v=1: 26,4,5,6,7,2,3,28,29,30,31,12,13,8,9,10,11,16,17,18,33,32,15$, $14,19,20,25,24,23,22,21,34,35,27,0$

- $v=2: 12,31,32,15,14,13,8,7,6,5,10,9,30,29,28,3,4,26,25,24,23,1,0$, $27,35,34,33,18,19,20,21,22,17,16,11$

- $v=3: 26,4,5,6,7,2,1,23,22,21,20,25,24,35,34,33,32,15,14,19,18,17$, $16,11,10,9,8,13,12,31,30,29,28,27,0$

- $v=4: 26,25,20,19,18,17,22,21,34,33,32,31,12,13,14,15,16,11,10,5,6$, $7,8,9,30,29,28,3,2,1,23,24,35,27,0$

- $v=5: 26,4,3,2,1,23,22,21,20,25,24,35,34,33,32,15,14,19,18,17,16,11$, $10,9,30,31,12,13,8,7,6,29,28,27,0$

- $v=6: 26,25,20,19,18,17,22,21,34,33,32,31,12,13,14,15,16,11,10,5,4$, $3,2,7,8,9,30,29,28,27,35,24,23,1,0$

- $v=7: 26,4,5,6,29,30,31,12,13,8,9,10,11,16,17,18,19,14,15,32,33,34$, $35,24,25,20,21,22,23,1,2,3,28,27,0$

- $v=8: 26,25,20,19,18,17,22,21,34,33,32,31,12,13,14,15,16,11,10,9,30$, $29,28,3,4,5,6,7,2,1,23,24,35,27,0$

- $v=9: 26,4,3,2,1,23,22,21,20,25,24,35,34,33,32,15,14,19,18,17,16,11$, $10,5,6,7,8,13,12,31,30,29,28,27,0$

- $v=10: 12,13,14,15,32,31,30,9,8,7,2,3,28,29,6,5,4,26,25,24,23,1,0$, $27,35,34,33,18,19,20,21,22,17,16,11$

- $v=11: 26,4,3,2,1,23,22,21,34,35,24,25,20,19,14,15,16,17,18,33,32$, $31,12,13,8,7,6,5,10,9,30,29,28,27,0$

- $v=12: 26,25,20,19,18,17,22,21,34,33,32,31,30,9,8,13,14,15,16,11,10$, $5,4,3,2,7,6,29,28,27,35,24,23,1,0$

- $v=13: 12,31,30,29,6,5,10,9,8,7,2,1,0,27,28,3,4,26,25,20,21,22,23$, $24,35,34,33,32,15,14,19,18,17,16,11$

- $v=14: 26,25,20,19,18,17,22,21,34,33,32,15,16,11,10,9,8,13,12,31,30$, $29,28,3,4,5,6,7,2,1,23,24,35,27,0$

- $v=15: 26,4,3,2,1,23,22,21,20,25,24,35,34,33,32,31,12,13,14,19,18$, $17,16,11,10,5,6,7,8,9,30,29,28,27,0$

- $v=16: 12,13,8,7,2,3,28,29,6,5,4,26,25,24,23,1,0,27,35,34,33,18,17$, $22,21,20,19,14,15,32,31,30,9,10,11$

- $v=17: 26,4,3,2,1,23,22,21,20,25,24,35,34,33,18,19,14,13,12,31,32$, $15,16,11,10,5,6,7,8,9,30,29,28,27,0$ 
- $v=18: 26,25,20,19,14,15,32,33,34,21,22,17,16,11,10,9,8,13,12,31,30$, $29,28,3,4,5,6,7,2,1,23,24,35,27,0$

- $v=19: 26,4,3,28,27,35,34,21,20,25,24,23,22,17,18,33,32,31,12,13,14$, $15,16,11,10,5,6,29,30,9,8,7,2,1,0$

- $v=20: 26,25,24,35,27,28,29,6,5,4,3,2,7,8,13,12,31,30,9,10,11,16,17$, $18,19,14,15,32,33,34,21,22,23,1,0$

- $v=21: 26,4,3,2,1,23,22,17,18,19,20,25,24,35,34,33,32,31,12,13,14$, $15,16,11,10,5,6,7,8,9,30,29,28,27,0$

- $v=22: 26,25,20,21,34,33,32,15,14,19,18,17,16,11,10,9,8,13,12,31,30$, $29,28,3,4,5,6,7,2,1,23,24,35,27,0$

- $v=23: 26,4,3,28,27,35,24,25,20,19,18,17,22,21,34,33,32,31,12,13,14$, $15,16,11,10,5,6,29,30,9,8,7,2,1,0$

- $v=24: 26,25,20,19,14,15,32,33,18,17,16,11,10,9,8,13,12,31,30,29,28$, $3,4,5,6,7,2,1,23,22,21,34,35,27,0$

- $v=25: 26,4,3,2,1,23,24,35,34,33,18,17,22,21,20,19,14,13,12,31,32$, $15,16,11,10,5,6,7,8,9,30,29,28,27,0$

- $v=26: 12,13,8,7,2,3,4,5,6,29,28,27,0,1,23,22,21,34,35,24,25,20,19$, $14,15,16,17,18,33,32,31,30,9,10,11$

- $v=27: 26,4,5,6,7,2,3,28,29,30,31,12,13,8,9,10,11,16,17,18,19,14,15$, $32,33,34,35,24,25,20,21,22,23,1,0$

- $v=28: 12,13,14,15,32,31,30,29,6,5,10,9,8,7,2,3,4,26,25,24,23,1,0$, $27,35,34,33,18,19,20,21,22,17,16,11$

- $v=29: 26,4,5,6,7,8,13,12,31,30,9,10,11,16,17,18,19,14,15,32,33,34$, $35,24,25,20,21,22,23,1,2,3,28,27,0$

- $v=30: 26,25,20,19,18,17,22,21,34,33,32,31,12,13,14,15,16,11,10,9,8$, $7,2,3,4,5,6,29,28,27,35,24,23,1,0$

- $v=31: 12,13,8,7,6,5,10,9,30,29,28,27,0,1,2,3,4,26,25,20,21,22,23$, $24,35,34,33,32,15,14,19,18,17,16,11$

- $v=32: 26,25,24,23,1,2,7,6,5,4,3,28,29,30,31,12,13,8,9,10,11,16,15$, $14,19,20,21,22,17,18,33,34,35,27,0$

- $v=33: 26,4,3,28,27,35,34,21,20,25,24,23,22,17,18,19,14,13,12,31,32$, $15,16,11,10,5,6,29,30,9,8,7,2,1,0$

- $v=34: 26,25,24,35,27,28,29,6,5,4,3,2,7,8,13,12,31,30,9,10,11,16,17$, $18,33,32,15,14,19,20,21,22,23,1,0$

- $v=35: 26,4,3,2,1,23,24,25,20,19,18,17,22,21,34,33,32,31,12,13,14$, $15,16,11,10,5,6,7,8,9,30,29,28,27,0$

\section{Proof that $b_{0} c_{0}$ and $b_{4} c_{4}$ are suitable edges for $J_{11}$}

Figure 2 shows the flower snark $J_{11}$ and here $b_{0} c_{0}$ and $b_{4} c_{4}$ correspond to the edges $(0,32)$ and $(11,12)$, respectively.

The pairs $(0,11),(0,12),(32,11)$, and $(32,12)$ are good in $J_{11}-\{(0,32),(11,12)\}$ due to the following hamiltonian paths, respectively: 
- $11,10,5,6,7,8,9,36,35,34,33,43,30,29,28,23,22,17,16,15,38,37,12,13$, $14,19,18,39,40,21,20,25,24,41,42,27,26,31,32,4,3,2,1,0$

- $12,13,14,15,16,11,10,5,4,32,31,26,25,24,41,42,27,28,23,22,17,18,19$, $20,21,40,39,38,37,36,9,8,7,6,35,34,3,2,1,29,30,43,33,0$

- $11,10,5,4,3,2,1,0,33,34,35,6,7,8,9,36,37,12,13,14,19,18,17,16,15,38$, $39,40,41,24,25,20,21,22,23,28,29,30,43,42,27,26,31,32$

- $12,13,8,7,2,3,4,5,6,35,34,33,0,1,29,28,23,22,17,18,39,38,37,36,9,10$, $11,16,15,14,19,20,21,40,41,24,25,26,27,42,43,30,31,32$

We have that $((0,32),(11,12))$ is good in $J_{11}-\{(0,32),(11,12)\}$ due to the following two disjoint paths with end-vertices 0 and 32 , and 11 and 12 , respectively, which together span $J_{11}$.

- $32,31,26,25,20,19,14,13,8,7,2,1,0$

- $12,37,38,15,16,17,18,39,40,21,22,23,24,41,42,27,28,29,30,43,33,34,3$, $4,5,6,35,36,9,10,11$

The following hamiltonian paths show that at least one of $(0,32)$ or $(11,12)$ is good in $J_{11}-\{v,(0,32),(11,12)\}$ for every $v \in V\left(J_{11}\right)$.

- $v=0: 12,13,14,15,38,37,36,35,6,5,10,9,8,7,2,1,29,30,31,32,4,3,34$, $33,43,42,41,24,23,28,27,26,25,20,19,18,39,40,21,22,17,16,11$

- $v=1: 32,4,5,6,7,2,3,34,35,36,37,12,13,8,9,10,11,16,17,18,19,14,15$, $38,39,40,41,24,23,22,21,20,25,26,31,30,29,28,27,42,43,33,0$

- $v=2: 12,37,38,15,14,13,8,7,6,5,10,9,36,35,34,3,4,32,31,30,29,1,0$, $33,43,42,41,24,23,28,27,26,25,20,19,18,39,40,21,22,17,16,11$

- $v=3: 32,4,5,6,7,2,1,29,28,27,26,31,30,43,42,41,40,21,22,23,24,25$, $20,19,14,15,38,39,18,17,16,11,10,9,8,13,12,37,36,35,34,33,0$

- $v=4: 32,31,26,25,24,23,28,27,42,41,40,39,18,17,22,21,20,19,14,13$, $12,37,38,15,16,11,10,5,6,7,8,9,36,35,34,3,2,1,29,30,43,33,0$

- $v=5: 32,4,3,2,1,29,28,27,26,31,30,43,42,41,40,21,22,23,24,25,20,19$, $14,15,38,39,18,17,16,11,10,9,36,37,12,13,8,7,6,35,34,33,0$

- $v=6: 32,31,26,25,24,23,28,27,42,41,40,39,18,17,22,21,20,19,14,13$, $12,37,38,15,16,11,10,5,4,3,2,7,8,9,36,35,34,33,43,30,29,1,0$

- $v=7: 32,4,5,6,35,36,37,12,13,8,9,10,11,16,17,18,19,14,15,38,39,40$, $41,24,23,22,21,20,25,26,31,30,29,28,27,42,43,33,34,3,2,1,0$

- $v=8: 32,31,26,25,24,23,28,27,42,41,40,39,18,17,22,21,20,19,14,13$, $12,37,38,15,16,11,10,9,36,35,34,3,4,5,6,7,2,1,29,30,43,33,0$

- $v=9: 32,4,3,2,1,29,28,27,26,31,30,43,42,41,40,21,22,23,24,25,20,19$, $14,15,38,39,18,17,16,11,10,5,6,7,8,13,12,37,36,35,34,33,0$

- $v=10: 12,13,14,15,38,37,36,9,8,7,2,3,34,35,6,5,4,32,31,30,29,1,0$, $33,43,42,41,24,23,28,27,26,25,20,19,18,39,40,21,22,17,16,11$

- $v=11: 32,4,3,2,1,29,28,27,26,31,30,43,42,41,40,21,20,25,24,23,22$, $17,16,15,14,19,18,39,38,37,12,13,8,7,6,5,10,9,36,35,34,33,0$ 
- $v=12: 32,31,26,25,24,23,28,27,42,41,40,39,18,17,22,21,20,19,14,13$, $8,9,10,11,16,15,38,37,36,35,34,3,4,5,6,7,2,1,29,30,43,33,0$

- $v=13: 12,37,36,35,6,5,10,9,8,7,2,1,0,33,34,3,4,32,31,26,27,28,29$, $30,43,42,41,40,21,22,23,24,25,20,19,14,15,38,39,18,17,16,11$

- $v=14: 32,31,26,25,24,41,42,27,28,23,22,17,18,19,20,21,40,39,38,15$, $16,11,10,9,8,13,12,37,36,35,34,3,4,5,6,7,2,1,29,30,43,33,0$

- $v=15: 32,4,3,2,1,29,28,27,26,31,30,43,42,41,40,21,22,23,24,25,20$, $19,14,13,12,37,38,39,18,17,16,11,10,5,6,7,8,9,36,35,34,33,0$

- $v=16: 12,13,8,7,2,3,34,35,6,5,4,32,31,30,29,1,0,33,43,42,41,24,25$, $26,27,28,23,22,17,18,39,40,21,20,19,14,15,38,37,36,9,10,11$

- $v=17: 32,4,3,2,1,29,28,27,26,31,30,43,42,41,40,21,22,23,24,25,20$, $19,18,39,38,37,12,13,14,15,16,11,10,5,6,7,8,9,36,35,34,33,0$

- $v=18: 32,31,26,25,24,23,28,27,42,41,40,39,38,15,14,19,20,21,22,17$, $16,11,10,9,8,13,12,37,36,35,34,3,4,5,6,7,2,1,29,30,43,33,0$

- $v=19: 32,4,3,2,1,29,28,27,26,31,30,43,42,41,40,21,20,25,24,23,22$, $17,18,39,38,37,12,13,14,15,16,11,10,5,6,7,8,9,36,35,34,33,0$

- $v=20: 32,31,26,25,24,41,42,27,28,23,22,21,40,39,38,15,14,19,18,17$, $16,11,10,9,8,13,12,37,36,35,34,3,4,5,6,7,2,1,29,30,43,33,0$

- $v=21: 32,4,3,2,1,29,28,27,26,31,30,43,42,41,40,39,18,17,22,23,24$, $25,20,19,14,13,12,37,38,15,16,11,10,5,6,7,8,9,36,35,34,33,0$

- $v=22: 32,31,26,25,24,23,28,27,42,41,40,21,20,19,14,15,38,39,18,17$, $16,11,10,9,8,13,12,37,36,35,34,3,4,5,6,7,2,1,29,30,43,33,0$

- $v=23: 32,4,3,2,1,29,28,27,26,31,30,43,42,41,24,25,20,19,18,17,22$, $21,40,39,38,37,12,13,14,15,16,11,10,5,6,7,8,9,36,35,34,33,0$

- $v=24: 32,31,26,25,20,21,22,23,28,27,42,41,40,39,38,15,14,19,18,17$, $16,11,10,9,8,13,12,37,36,35,34,3,4,5,6,7,2,1,29,30,43,33,0$

- $v=25: 32,4,3,2,1,29,28,27,26,31,30,43,42,41,24,23,22,17,18,19,20$, $21,40,39,38,37,12,13,14,15,16,11,10,5,6,7,8,9,36,35,34,33,0$

- $v=26$ : 32, 31, 30, 29, 1, 2, 7, 6, 5, 4, 3, 34, 35, 36, 37, 12, 13, 8, 9, 10, 11, 16, 17, $18,19,14,15,38,39,40,41,24,25,20,21,22,23,28,27,42,43,33,0$

- $v=27: 32,4,3,2,1,29,28,23,24,25,26,31,30,43,42,41,40,39,18,17,22$, $21,20,19,14,13,12,37,38,15,16,11,10,5,6,7,8,9,36,35,34,33,0$

- $v=28: 32,31,26,27,42,41,40,21,22,23,24,25,20,19,14,15,38,39,18,17$, $16,11,10,9,8,13,12,37,36,35,34,3,4,5,6,7,2,1,29,30,43,33,0$

- $v=29: 32,4,3,34,33,43,30,31,26,25,24,23,28,27,42,41,40,39,18,17,22$, $21,20,19,14,13,12,37,38,15,16,11,10,5,6,35,36,9,8,7,2,1,0$

- $v=30: 32,31,26,25,20,21,22,23,24,41,40,39,38,15,14,19,18,17,16,11$, $10,9,8,13,12,37,36,35,34,3,4,5,6,7,2,1,29,28,27,42,43,33,0$

- $v=31: 32,4,3,2,1,29,30,43,42,41,24,23,28,27,26,25,20,19,18,17,22$, $21,40,39,38,37,12,13,14,15,16,11,10,5,6,7,8,9,36,35,34,33,0$ 
- $v=32: 12,13,8,7,2,3,4,5,6,35,34,33,0,1,29,28,27,26,31,30,43,42,41$, $40,21,20,25,24,23,22,17,16,15,14,19,18,39,38,37,36,9,10,11$

- $v=33: 32,4,5,6,7,2,3,34,35,36,37,12,13,8,9,10,11,16,17,18,19,14,15$, $38,39,40,41,24,23,22,21,20,25,26,31,30,43,42,27,28,29,1,0$

- $v=34: 12,13,14,15,38,37,36,35,6,5,10,9,8,7,2,3,4,32,31,30,29,1,0$, $33,43,42,41,24,23,28,27,26,25,20,19,18,39,40,21,22,17,16,11$

- $v=35: 32,4,5,6,7,8,13,12,37,36,9,10,11,16,17,18,19,14,15,38,39,40$, $41,24,23,22,21,20,25,26,31,30,29,28,27,42,43,33,34,3,2,1,0$

- $v=36: 32,31,26,25,24,23,28,27,42,41,40,39,18,17,22,21,20,19,14,13$, $12,37,38,15,16,11,10,9,8,7,2,3,4,5,6,35,34,33,43,30,29,1,0$

- $v=37: 12,13,8,7,6,5,10,9,36,35,34,33,0,1,2,3,4,32,31,26,27,28,29$, $30,43,42,41,40,21,22,23,24,25,20,19,14,15,38,39,18,17,16,11$

- $v=38: 32,31,26,25,24,23,28,27,42,41,40,39,18,17,22,21,20,19,14,15$, $16,11,10,9,8,13,12,37,36,35,34,3,4,5,6,7,2,1,29,30,43,33,0$

- $v=39: 32,4,3,2,1,29,28,27,26,31,30,43,42,41,40,21,20,25,24,23,22$, $17,18,19,14,13,12,37,38,15,16,11,10,5,6,7,8,9,36,35,34,33,0$

- $v=40: 32,31,26,25,24,41,42,27,28,23,22,21,20,19,14,15,38,39,18,17$, $16,11,10,9,8,13,12,37,36,35,34,3,4,5,6,7,2,1,29,30,43,33,0$

- $v=41: 32,4,3,2,1,29,28,27,42,43,30,31,26,25,24,23,22,17,18,19,20$, $21,40,39,38,37,12,13,14,15,16,11,10,5,6,7,8,9,36,35,34,33,0$

- $v=42: 32,31,26,27,28,23,22,21,20,25,24,41,40,39,38,15,14,19,18,17$, $16,11,10,9,8,13,12,37,36,35,34,3,4,5,6,7,2,1,29,30,43,33,0$

- $v=43: 32,4,3,2,1,29,30,31,26,25,24,23,28,27,42,41,40,39,18,17,22$, $21,20,19,14,13,12,37,38,15,16,11,10,5,6,7,8,9,36,35,34,33,0$

\section{Proof that $b_{0} c_{0}$ and $b_{4} c_{4}$ are suitable edges for $J_{13}$}

Figure 3 shows the flower snark $J_{13}$ and here $b_{0} c_{0}$ and $b_{4} c_{4}$ correspond to the edges $(0,38)$ and $(11,12)$, respectively.

The pairs $(0,11),(0,12),(38,11)$, and $(38,12)$ are good in $J_{13}-\{(0,38),(11,12)\}$ due to the following hamiltonian paths, respectively:

- $11,10,5,6,7,8,9,42,41,40,39,51,36,35,34,29,28,23,22,17,16,15,44,43$, $12,13,14,19,18,45,46,21,20,25,24,47,48,27,26,31,30,49,50,33,32,37$, $38,4,3,2,1,0$

- $12,13,14,15,16,11,10,5,4,38,37,32,31,30,29,34,33,50,49,48,47,24,23$, $28,27,26,25,20,19,18,17,22,21,46,45,44,43,42,9,8,7,6,41,40,3,2,1,35$, $36,51,39,0$

- $11,10,5,4,3,2,1,0,39,40,41,6,7,8,9,42,43,12,13,14,19,18,17,16,15,44$, $45,46,47,24,23,22,21,20,25,26,31,30,29,28,27,48,49,50,51,36,35,34$, $33,32,37,38$

- $12,13,8,7,2,3,4,5,6,41,40,39,0,1,35,34,29,28,23,22,17,18,45,44,43$, $42,9,10,11,16,15,14,19,20,21,46,47,24,25,26,27,48,49,30,31,32,33,50$, $51,36,37,38$ 
The pair of pairs $((0,38),(11,12))$ is good in $J_{13}-\{(0,38),(11,12)\}$ due to the following two disjoint paths with end-vertices 0 and 38 , and 11 and 12 , respectively, which together $\operatorname{span} J_{13}$.

- $38,37,32,31,26,25,20,19,14,13,8,7,2,1,0$

- $12,43,44,15,16,17,18,45,46,21,22,23,24,47,48,27,28,29,30,49,50,33$, $34,35,36,51,39,40,3,4,5,6,41,42,9,10,11$

The following hamiltonian paths show that at least one of $(0,38)$ or $(11,12)$ is good in $J_{13}-\{v,(0,38),(11,12)\}$ for every $v \in V\left(J_{13}\right)$.

- $v=0: 12,13,14,15,44,43,42,41,6,5,10,9,8,7,2,1,35,36,37,38,4,3,40$, $39,51,50,49,30,29,34,33,32,31,26,25,24,23,28,27,48,47,46,45,18,19$, $20,21,22,17,16,11$

- $v=1: 38,4,5,6,7,2,3,40,41,42,43,12,13,8,9,10,11,16,17,18,19,14,15$, $44,45,46,47,24,23,22,21,20,25,26,31,30,49,48,27,28,29,34,35,36,37$, $32,33,50,51,39,0$

- $v=2: 12,43,44,15,14,13,8,7,6,5,10,9,42,41,40,3,4,38,37,36,35,1,0$, $39,51,50,49,30,29,34,33,32,31,26,25,24,23,28,27,48,47,46,45,18,19$, $20,21,22,17,16,11$

- $v=3: 38,4,5,6,7,2,1,35,34,33,32,37,36,51,50,49,48,27,26,31,30,29$, $28,23,22,21,20,25,24,47,46,45,44,15,14,19,18,17,16,11,10,9,8,13,12$, $43,42,41,40,39,0$

- $v=4: 38,37,32,31,30,29,34,33,50,49,48,47,24,23,28,27,26,25,20,19$, $18,17,22,21,46,45,44,43,12,13,14,15,16,11,10,5,6,7,8,9,42,41,40,3,2$, $1,35,36,51,39,0$

- $v=5: 38,4,3,2,1,35,34,33,32,37,36,51,50,49,48,27,26,31,30,29,28,23$, $22,21,20,25,24,47,46,45,44,15,14,19,18,17,16,11,10,9,42,43,12,13,8$, $7,6,41,40,39,0$

- $v=6: 38,37,32,31,30,29,34,33,50,49,48,47,24,23,28,27,26,25,20,19$, $18,17,22,21,46,45,44,43,12,13,14,15,16,11,10,5,4,3,2,7,8,9,42,41,40$, $39,51,36,35,1,0$

- $v=7: 38,4,5,6,41,42,43,12,13,8,9,10,11,16,17,18,19,14,15,44,45,46$, $47,24,23,22,21,20,25,26,31,30,29,28,27,48,49,50,51,36,37,32,33,34$, $35,1,2,3,40,39,0$

- $v=8: 38,37,32,31,30,29,34,33,50,49,48,47,24,23,28,27,26,25,20,19$, $18,17,22,21,46,45,44,43,12,13,14,15,16,11,10,9,42,41,40,3,4,5,6,7,2$, $1,35,36,51,39,0$

- $v=9: 38,4,3,2,1,35,34,33,32,37,36,51,50,49,48,27,26,31,30,29,28,23$, $22,21,20,25,24,47,46,45,44,15,14,19,18,17,16,11,10,5,6,7,8,13,12,43$, $42,41,40,39,0$

- $v=10: 12,13,14,15,44,43,42,9,8,7,2,3,40,41,6,5,4,38,37,36,35,1,0$, $39,51,50,49,30,29,34,33,32,31,26,25,24,23,28,27,48,47,46,45,18,19$, $20,21,22,17,16,11$ 
- $v=11: 38,4,3,2,1,35,34,33,32,37,36,51,50,49,48,27,26,31,30,29,28$, $23,22,21,46,47,24,25,20,19,14,15,16,17,18,45,44,43,12,13,8,7,6,5,10$, $9,42,41,40,39,0$

- $v=12: 38,37,32,31,30,29,34,33,50,49,48,47,24,23,28,27,26,25,20,19$, $18,17,22,21,46,45,44,43,42,9,8,13,14,15,16,11,10,5,4,3,2,7,6,41,40$, $39,51,36,35,1,0$

- $v=13: 12,43,42,41,6,5,10,9,8,7,2,1,0,39,40,3,4,38,37,32,33,34,35$, $36,51,50,49,48,27,26,31,30,29,28,23,22,21,20,25,24,47,46,45,44,15$, $14,19,18,17,16,11$

- $v=14: 38,37,32,31,30,29,34,33,50,49,48,47,24,23,28,27,26,25,20,19$, $18,17,22,21,46,45,44,15,16,11,10,9,8,13,12,43,42,41,40,3,4,5,6,7,2$, $1,35,36,51,39,0$

- $v=15: 38,4,3,2,1,35,34,33,32,37,36,51,50,49,48,27,26,31,30,29,28$, $23,22,21,20,25,24,47,46,45,44,43,12,13,14,19,18,17,16,11,10,5,6,7,8$, $9,42,41,40,39,0$

- $v=16: 12,13,8,7,2,3,40,41,6,5,4,38,37,36,35,1,0,39,51,50,49,30,29$, $34,33,32,31,26,25,24,23,28,27,48,47,46,45,18,17,22,21,20,19,14,15$, $44,43,42,9,10,11$

- $v=17: 38,4,3,2,1,35,34,33,32,37,36,51,50,49,48,27,26,31,30,29,28$, $23,22,21,20,25,24,47,46,45,18,19,14,13,12,43,44,15,16,11,10,5,6,7,8$, $9,42,41,40,39,0$

- $v=18: 38,37,32,31,30,29,34,33,50,49,48,47,24,23,28,27,26,25,20,19$, $14,15,44,45,46,21,22,17,16,11,10,9,8,13,12,43,42,41,40,3,4,5,6,7,2$, $1,35,36,51,39,0$

- $v=19: 38,4,3,2,1,35,34,33,32,37,36,51,50,49,48,27,28,29,30,31,26$, $25,20,21,46,47,24,23,22,17,18,45,44,43,12,13,14,15,16,11,10,5,6,7,8$, $9,42,41,40,39,0$

- $v=20: 38,37,32,31,30,29,34,33,50,49,48,47,24,25,26,27,28,23,22,21$, $46,45,44,15,14,19,18,17,16,11,10,9,8,13,12,43,42,41,40,3,4,5,6,7,2$, $1,35,36,51,39,0$

- $v=21: 38,4,3,2,1,35,34,33,32,37,36,51,50,49,48,27,26,31,30,29,28$, $23,22,17,18,19,20,25,24,47,46,45,44,43,12,13,14,15,16,11,10,5,6,7,8$, $9,42,41,40,39,0$

- $v=22: 38,37,32,31,30,29,34,33,50,49,48,47,24,23,28,27,26,25,20,21$, $46,45,44,15,14,19,18,17,16,11,10,9,8,13,12,43,42,41,40,3,4,5,6,7,2$, $1,35,36,51,39,0$

- $v=23: 38,4,3,2,1,35,34,33,32,37,36,51,50,49,48,27,28,29,30,31,26$, $25,24,47,46,45,18,17,22,21,20,19,14,13,12,43,44,15,16,11,10,5,6,7,8$, $9,42,41,40,39,0$

- $v=24: 38,37,32,31,30,29,34,33,50,49,48,47,46,21,22,23,28,27,26,25$, $20,19,14,15,44,45,18,17,16,11,10,9,8,13,12,43,42,41,40,3,4,5,6,7,2$, $1,35,36,51,39,0$ 
- $v=25: 38,4,3,2,1,35,34,33,32,37,36,51,50,49,48,27,26,31,30,29,28$, $23,24,47,46,45,18,17,22,21,20,19,14,13,12,43,44,15,16,11,10,5,6,7,8$, $9,42,41,40,39,0$

- $v=26: 38,37,32,31,30,29,34,33,50,49,48,27,28,23,22,21,20,25,24,47$, $46,45,44,15,14,19,18,17,16,11,10,9,8,13,12,43,42,41,40,3,4,5,6,7,2$, $1,35,36,51,39,0$

- $v=27: 38,4,3,2,1,35,34,33,32,37,36,51,50,49,48,47,24,23,28,29,30$, $31,26,25,20,19,18,17,22,21,46,45,44,43,12,13,14,15,16,11,10,5,6,7,8$, $9,42,41,40,39,0$

- $v=28: 38,37,32,31,30,29,34,33,50,49,48,27,26,25,20,21,22,23,24,47$, $46,45,44,15,14,19,18,17,16,11,10,9,8,13,12,43,42,41,40,3,4,5,6,7,2$, $1,35,36,51,39,0$

- $v=29: 38,4,3,2,1,35,34,33,32,37,36,51,50,49,30,31,26,25,24,23,28$, $27,48,47,46,45,18,17,22,21,20,19,14,13,12,43,44,15,16,11,10,5,6,7,8$, $9,42,41,40,39,0$

- $v=30: 38,37,32,31,26,27,28,29,34,33,50,49,48,47,46,21,22,23,24,25$, $20,19,14,15,44,45,18,17,16,11,10,9,8,13,12,43,42,41,40,3,4,5,6,7,2$, $1,35,36,51,39,0$

- $v=31: 38,4,3,2,1,35,34,33,32,37,36,51,50,49,30,29,28,23,24,25,26$, $27,48,47,46,45,18,17,22,21,20,19,14,13,12,43,44,15,16,11,10,5,6,7,8$, $9,42,41,40,39,0$

- $v=32: 38,37,36,35,1,2,7,6,5,4,3,40,41,42,43,12,13,8,9,10,11,16,17$, $18,19,14,15,44,45,46,47,24,23,22,21,20,25,26,31,30,49,48,27,28,29$, $34,33,50,51,39,0$

- $v=33: 38,4,3,2,1,35,34,29,30,31,32,37,36,51,50,49,48,47,24,23,28$, $27,26,25,20,19,18,17,22,21,46,45,44,43,12,13,14,15,16,11,10,5,6,7,8$, $9,42,41,40,39,0$

- $v=34: 38,37,32,33,50,49,48,27,26,31,30,29,28,23,22,21,20,25,24,47$, $46,45,44,15,14,19,18,17,16,11,10,9,8,13,12,43,42,41,40,3,4,5,6,7,2$, $1,35,36,51,39,0$

- $v=35: 38,4,3,40,39,51,36,37,32,31,30,29,34,33,50,49,48,47,24,23,28$, $27,26,25,20,19,18,17,22,21,46,45,44,43,12,13,14,15,16,11,10,5,6,41$, $42,9,8,7,2,1,0$

- $v=36$ : 38, 37, 32, 31, 26, 27, 28, 29, 30, 49, 48, 47, 46, 21, 22, 23, 24, 25, 20, 19, $14,15,44,45,18,17,16,11,10,9,8,13,12,43,42,41,40,3,4,5,6,7,2,1,35$, $34,33,50,51,39,0$

- $v=37: 38,4,3,2,1,35,36,51,50,49,30,29,34,33,32,31,26,25,24,23,28$, $27,48,47,46,45,18,17,22,21,20,19,14,13,12,43,44,15,16,11,10,5,6,7,8$, $9,42,41,40,39,0$

- $v=38: 12,13,8,7,2,3,4,5,6,41,40,39,0,1,35,34,33,32,37,36,51,50,49$, $48,27,26,31,30,29,28,23,22,21,46,47,24,25,20,19,14,15,16,17,18,45$, $44,43,42,9,10,11$ 
- $v=39: 38,4,5,6,7,2,3,40,41,42,43,12,13,8,9,10,11,16,17,18,19,14,15$, $44,45,46,47,24,23,22,21,20,25,26,31,30,29,28,27,48,49,50,51,36,37$, $32,33,34,35,1,0$

- $v=40: 12,13,14,15,44,43,42,41,6,5,10,9,8,7,2,3,4,38,37,36,35,1,0$, $39,51,50,49,30,29,34,33,32,31,26,25,24,23,28,27,48,47,46,45,18,19$, $20,21,22,17,16,11$

- $v=41: 38,4,5,6,7,8,13,12,43,42,9,10,11,16,17,18,19,14,15,44,45,46$, $47,24,23,22,21,20,25,26,31,30,29,28,27,48,49,50,51,36,37,32,33,34$, $35,1,2,3,40,39,0$

- $v=42: 38,37,32,31,30,29,34,33,50,49,48,47,24,23,28,27,26,25,20,19$, $18,17,22,21,46,45,44,43,12,13,14,15,16,11,10,9,8,7,2,3,4,5,6,41,40$, $39,51,36,35,1,0$

- $v=43: 12,13,8,7,6,5,10,9,42,41,40,39,0,1,2,3,4,38,37,32,33,34,35$, $36,51,50,49,48,27,26,31,30,29,28,23,22,21,20,25,24,47,46,45,44,15$, $14,19,18,17,16,11$

- $v=44: 38,37,32,31,30,29,34,33,50,49,48,47,24,25,26,27,28,23,22,17$, $18,45,46,21,20,19,14,15,16,11,10,9,8,13,12,43,42,41,40,3,4,5,6,7,2$, $1,35,36,51,39,0$

- $v=45: 38,4,3,2,1,35,34,33,32,37,36,51,50,49,48,27,28,29,30,31,26$, $25,20,21,46,47,24,23,22,17,18,19,14,13,12,43,44,15,16,11,10,5,6,7,8$, $9,42,41,40,39,0$

- $v=46: 38,37,32,31,30,29,34,33,50,49,48,47,24,25,26,27,28,23,22,21$, $20,19,14,15,44,45,18,17,16,11,10,9,8,13,12,43,42,41,40,3,4,5,6,7,2$, $1,35,36,51,39,0$

- $v=47: 38,4,3,2,1,35,34,33,32,37,36,51,50,49,48,27,26,31,30,29,28$, $23,24,25,20,19,18,17,22,21,46,45,44,43,12,13,14,15,16,11,10,5,6,7,8$, $9,42,41,40,39,0$

- $v=48: 38,37,32,31,30,49,50,33,34,29,28,27,26,25,20,21,22,23,24,47$, $46,45,44,15,14,19,18,17,16,11,10,9,8,13,12,43,42,41,40,3,4,5,6,7,2$, $1,35,36,51,39,0$

- $v=49: 38,4,3,2,1,35,34,33,50,51,36,37,32,31,30,29,28,23,24,25,26$, $27,48,47,46,45,18,17,22,21,20,19,14,13,12,43,44,15,16,11,10,5,6,7,8$, $9,42,41,40,39,0$

- $v=50: 38,37,32,33,34,29,28,27,26,31,30,49,48,47,46,21,22,23,24,25$, $20,19,14,15,44,45,18,17,16,11,10,9,8,13,12,43,42,41,40,3,4,5,6,7,2$, $1,35,36,51,39,0$

- $v=51: 38,4,3,2,1,35,36,37,32,31,30,29,34,33,50,49,48,47,24,23,28$, $27,26,25,20,19,18,17,22,21,46,45,44,43,12,13,14,15,16,11,10,5,6,7,8$, 9, 42, 41, 40, 39, 0 